\title{
Penetrating Head Injury in Young Adulthood Exacerbates Cognitive Decline in Later Years
}

\author{
Suzanne Corkin, T. John Rosen, Edith V. Sullivan, ${ }^{a}$ and Rae Ann Clegg \\ Department of Brain and Cognitive Sciences and Clinical Research Center, Massachusetts Institute of Technology, \\ Cambridge, Massachusetts 02139
}

\begin{abstract}
Few investigators have studied whether the behavioral effects of brain insult in adulthood are stable after the period of maximum recovery. We addressed this issue in a 30-year longitudinal study of 84 veterans of World War II, 57 with penetrating head injury $(\mathrm{HI})$ and 27 with peripheral nerve injury (PNI), matched with respect to age, premorbid intelligence, and premorbld education. Each subject was examined during the 1950s and during the 1980s; each examination included the largely verbal Army General Classification Test (AGCT) (with Vocabulary, Arithmetic, and Block Counting subscales) and the Hidden Figures Test (which measures figure-ground discrimination). $\mathrm{HI}$ exacerbated decline in performance over time, irrespective of lesion site or cognitive test. $\mathrm{HI}$ and PNI subjects differed significantly $(p<0.05)$ in AGCT Total and Arithmetic change scores, and means were in the same direction for all other measures. In analyses contrasting subjects in each of the eight lesion groups to PNI subjects, those with left parietal lobe injuries showed significantly greater decline from the 1950 s to the 1980s on the Vocabulary and Arithmetic subscales of the AGCT, as did those with left temporal lobe injuries on the Arithmetic subscale, whereas subjects with right parietal lobe injuries showed significantly greater decline on the Hidden Figures Test. We hypothesize that the observed reduction of cognitive capacities late in life was due to some combination of $\mathrm{Hl}$ in young adulthood, secondary effects of the injury occurring with time, effects of stress on remaining brain tissue caused by functioning for decades in a compromised state, and changes in the brain occurring
\end{abstract}

\footnotetext{
Received July 28, 1988; revised May 1, 1989; accepted May 1, 1989.

We acknowledge the seminal contributions of Hans-Lukas Teuber, Josephine Semmes, Sidney Weinstein, and Lila Ghent Braine, who carefully documented the effects of penetrating head injury in our subjects during the early 1950s. The raw data and publications from their study at New York University-Bellevue Medical Center contributed to the follow-up study and analyses reported here. The 1980 s evaluations were conducted at the MIT Clinical Rescarch Center, whosc staff provided superb patient care. We thank Max Rohrlich for his assistance in establishing our temporary laboratory in Manhattan. Other support came from USPHS grants MH 24433 and RR 00088 . We are grateful to Karen Shedlack and Allison Feeley for data collection, to Martha J. Ferry, Sasha Nyary, and Elliza McGrand for manuscript preparation and to Thomas J. Pincince, H. David Shea, and Joy Yucaitis for preparation of graphics. We also acknowledge insightful contributions to our thinking from Brenda Milner and Caleb E. Finch.

Correspondence should be addressed to Suzanne Corkin, Department of Brain and Cognitive Sciences, E10-003A, Massachusetts Institute of Technology, 77 Massachusetts Avenue, Cambridge, MA 02139.

- Present address: Psychiatry Service, V.A. Medical Center, Palo Alto, CA 94304 and Department of Psychiatry and Behavioral Science, Stanford University Medical Center, Stanford, CA 94305.

Copyright (C) 1989 Society for Neuroscience $0270-6474 / 89 / 113876-08 \$ 02.00 / 0$
}

with age. Although the HI subjects were not demented, follow-up studies must assess whether exacerbated decline is a harbinger of dementia.

Few studies have examined the concomitants of aging in a previously injured brain. This paper documents the effects of penetrating head injury (HI) on age-related cognitive decline over a 30 -year period. The fundamental question was whether cognitive performance would decline more over time in $\mathrm{HI}$ than in control subjects, i.e., whether HI subjects would manifest "exacerbated decline" of cognitive capacities.

We use the term "aging" to mean the alterations that characterize normal old age, which must be distinguished from the alterations secondary to $\mathrm{HI}$ that occur over time independently of aging. The mechanisms underlying the hypothesized interaction between aging and injury could result from morphological and chemical processes associated with advancing chronological age, from processes that are time-dependent rather than agedependent, or from both types of processes. If the interaction were age-related, we would expect the effects of $\mathrm{HI}$ on cognitive decline to stem from the combination of abnormalities due to aging and the primary and secondary consequences of injury. Alternatively, a time-dependent process, such as transneuronal degeneration (Cowan, 1970), emanating from the injured region, could also lead to further deterioration in a subject's clinical condition long after the time of injury (Geschwind, 1974).

In addition to determining whether prior $\mathrm{HI}$ exacerbated cognitive decline in later years, we sought to determine which lesions exacerbated decline of which cognitive capacities. If most capacities showed exacerbated decline following injury to any site, it would imply that the effect was generalized. In contrast, if exacerbated decline were restricted to specific combinations of a cognitive capacity and a lesion site, it would imply that the effect was focal.

Our data regarding the interaction of aging (or time) and HI came from a longitudinal study that examined a group of 314 veterans who had sustained either a penetrating HI or a peripheral nerve injury (PNI) while serving in the armed forces during World War II. These men had been recruited and tested during the late 1940s and the 1950s by Teuber and his colleagues at New York University-Bellevue Medical Center. Potential subjects had been identified through a survey of Veterans Administration rosters of World War II veterans living near New York City who had received injuries to the brain or to the peripheral nervous system (Semmes et al., 1960). The HI and PNI subjects were exceptionally comparable in that all had served in the armed forces during World War II, had incurred injuries 
Table 1. Characteristics of subjects

\begin{tabular}{|c|c|c|c|c|c|c|c|}
\hline & & & & & Education & & \\
\hline & $\begin{array}{l}\text { Age at } \\
\text { injury }\end{array}$ & $\begin{array}{l}\text { Age at } \\
1950 \text { s test }\end{array}$ & $\begin{array}{l}\text { Age at } \\
1980 \text { s test }\end{array}$ & $\begin{array}{l}\text { Preinjury } \\
\text { AGCT }\end{array}$ & Preinjury & $\begin{array}{l}\text { Post- } \\
\text { injury }\end{array}$ & Total \\
\hline Head inj & $\imath=57)$ & & & & & & \\
\hline Mean & 23.9 & 32.2 & 61.9 & 110.4 & 11.5 & 0.7 & 12.2 \\
\hline Range & $18-33$ & $25-41$ & $54-72$ & $73-136$ & $6-16$ & $0-8$ & $6-20$ \\
\hline Peripher & ve injur & $=27)$ & & & & & \\
\hline Mean & 24.4 & 32.7 & 63.5 & 112.6 & 12.1 & 0.9 & 13.1 \\
\hline Range & $19-34$ & $27-42$ & $56-71$ & $79-141$ & $7-16$ & $0-5$ & $7-20$ \\
\hline
\end{tabular}

The groups did not differ significantly in mean on any of the above measures.

to the nervous system that had produced lasting impairment, and had been enrolled on Veterans Administration rosters.

Between 1979 and 1985 , our laboratory systematically retested 84 of the surviving veterans by repeating sensory, motor, and cognitive tests that had been administered in the 1950s at Bellevue Medical Center. Results from two cognitive tests are reported here.

\section{Materials and Methods}

Subjects. The experimental group comprised the 57 men with penetrating HI, and the control group comprised the 27 men with PNI who had been seen first in Teuber's New York laboratory and subsequently in our own. The ages of the subjects ranged from 19 to 34 years at the time of injury, from 25 to 42 at the 1950 s test, and from 54 to 72 at the 1980 s test (Table 1). The interval between the $1950 \mathrm{~s}$ and $1980 \mathrm{~s}$ tests was similar for the HI and PNI groups, 29.7 years (SD 2.0) for subjects with HI and 30.8 years (SD 1.7) for subjects with PNI.

The initial determinations of lesion location were established during subjects' 1950 s visits to the New York University-Bellevue Medical Center laboratory and were based on skull $x$-rays and surgeon's notes dating from the time of injury. Subsequently, CT scans were reviewed by neuroradiologists. Localization procedures were aided by the fact that the brain lesions were generally large and focal and by the fact that skull plates (when present) covered the regions incurring damage. Lesions were classified with respect to side of injury (left, right) and lobe (frontal, temporal, parietal, occipital), giving a total of eight possible lesion sites (Table 2). HI subjects were grouped according to whether their injuries invaded or spared each of the 8 sites. These subjects included 24 men presumed to have exclusively left-sided lesions, 24 presumed to have exclusively right-sided lesions, and 9 with bilateral lesions. None of the HI subgroups differed from the others nor from the PNI group in age at injury or age when tested, with one exception: Subjects with injuries of the right parietal lobe were on average 2.5 years younger at the time of injury and 3.1 years younger when seen in the 1980 s than the HI subjects whose lesions spared that site.

$\mathrm{HI}$ and PNI subjects received an average of 12 years of education prior to injury and less than 1 year subsequently (Table 1). Military records provided induction (and thus preinjury) Army General Classification Test (AGCT) Total scores for 42 of the 84 subjects. Analyses of induction AGCT Total scores and years of education before injury showed no differences between the HI and PNI groups (Table 1) and none among the 8 lesion groups.

Cognitive tests. Subjects received 2 pencil-and-paper tests, both timed. Four measures were obtained from the AGCT and one from the Hidden Figures Test. All test formats and procedures were the same at the 1950s and 1980s administrations (Teuber and Weinstein, 1956; Weinstein and Teuber, 1957). None of the scores was age-corrected.

Some subjects had hemianopias or smaller scotomata. Testing procedures were adapted as necessary so as to minimize the effects of such visual abnormalities on performance, e.g., by presenting stimuli and test materials in the intact field. All subjects' vision was adequate to perform all tests.

$A G C T$. The AGCT is a 40 -min test of overall intelligence that includes 3 types of items: Vocabulary, Arithmetic word problems, and Block Counting (Personnel Research Section, 1943). Separate subscores were calculated for each type of item, and the AGCT Total score was cal- culated from the entire set of items. Each subscore was defined as the number of correct items minus one-third the number of errors. As is customary, AGCT Total scores were transformed to a scale with a mean of 100 and an SD of 20 . The Total score roughly corresponds to a WAIS-R Full-Scale IQ rating, which has a mean of 100 and an SD of 15 (Wechsler, 1981).

Hidden Figures Test. The Hidden Figures Test was administered according to the general procedure outlined by Thurstone (1944). Subjects used a red felt-tip pen to trace a simple geometric figure that was embedded in a complex geometric figure. A subject's score was the total number of figures correctly traced out of 61 .

\section{Results}

Ten-year effects of head injury

To establish a reference point for evaluating subsequent change, $t$ tests compared the various lesion groups to the PNI group with respect to performance in the 1950 s (10 years after injury and thus presumably after the period of functional recovery). On all 5 cognitive measures (AGCT Total, Vocabulary, Arithmetic, Block Counting, Hidden Figures), the HI group was inferior to the PNI group at the 0.05 significance level or better (Table 3).

Ten years after injury, left hemisphere lesions had more deleterious effects on the AGCT and Hidden Figures Test than had right hemisphere lesions. Relative to PNI subjects, the only significant deficit following injury to the right hemisphere occurred on the Hidden Figures Test among subjects with right occipital lesions $(p=0.01)$. Among the subjects who received injuries to the left hemisphere, the left parietal and left occipital groups were significantly inferior to the PNI group on all five measures ( $p<0.05$ for each) (Table 4$)$. The small left temporal group also showed significant deficits relative to PNI subjects on AGCT Total, Arithmetic, and Block Counting ( $p \leq 0.05$ for each). The left frontal group, although consistently inferior to

Table 2. Site and side of cerebral injury in 57 World War II veterans

\begin{tabular}{lcc} 
& \multicolumn{2}{c}{ Side of injury } \\
\cline { 2 - 3 } Site of injury & Left & Right \\
\hline Frontal lobe & 15 & 12 \\
Temporal lobe & 5 & 10 \\
Parietal lobe & 14 & 13 \\
Occipital lobe & 7 & 9 \\
Cerebellum & 2 & 1
\end{tabular}

Entries in the Left and Right columns total more than the number of head-injured veterans (57) because many sustained injuries to multiple regions (mean number of injured lobes per veteran $=1.5$ ). 
Table 3. Test performance in head-injured and control groups as a function of time

\begin{tabular}{|c|c|c|c|c|c|c|c|}
\hline & \multicolumn{3}{|c|}{$1950 \mathrm{~s}$} & \multicolumn{2}{|l|}{$1980 \mathrm{~s}$} & \multicolumn{2}{|c|}{$\begin{array}{l}\text { 1980s-1950s } \\
\text { differences }\end{array}$} \\
\hline & $n^{a}$ & Mean & SD & Mean & $\mathrm{SD}$ & Mean & SD \\
\hline Head injury & 57 & & & & & & \\
\hline AGCT Total & 44 & 113.9 & 17.2 & 106.0 & 20.5 & $-7.9^{b}$ & 13.1 \\
\hline Vocabulary & 42 & 30.0 & 9.8 & 30.0 & 9.9 & 0.0 & 5.6 \\
\hline Arithmetic & 42 & 32.9 & 7.4 & 29.5 & 9.7 & $-3.4^{b}$ & 5.0 \\
\hline Block Counting & 42 & 28.9 & 7.4 & 24.3 & 10.0 & $-4.6^{h}$ & 8.6 \\
\hline Hidden Figures & 25 & 26.2 & 12.1 & 16.1 & 10.3 & $-10.1^{b}$ & 9.1 \\
\hline Peripheral nerve injury & 27 & & & & & & \\
\hline AGCT Total & 21 & 125.9 & 14.5 & 126.3 & 12.6 & 0.4 & 10.4 \\
\hline Vocabulary & 19 & 36.0 & 8.5 & 38.8 & 7.0 & $2.8^{c}$ & 5.4 \\
\hline Arithmetic & 19 & 38.9 & 6.8 & 38.9 & 5.6 & 0.0 & 6.0 \\
\hline Block Counting & 19 & 35.2 & 9.5 & 32.3 & 7.8 & $-2.9^{c}$ & 4.8 \\
\hline Hidden Figures & 14 & 35.4 & 12.3 & 27.9 & 11.6 & $-7.5^{c}$ & 9.8 \\
\hline
\end{tabular}

The Head Injury group was inferior to the Peripheral Nerve Injury group on each $1950 \mathrm{~s}$ test $(p \leq 0.05)$ and declined significantly more from the 1950s to the 1980s on AGCT Total and Arithmetic than did the Peripheral Nerve Injury group $(p \leq 0.05)$.

${ }^{a}$ In the 1950 s, some subjects received the AGCT but not the Hidden Figures Test, and vice versa.

"Differed from 0.0 at $p \leq 0.01$.

CDiffered from 0.0 at $p \leq 0.05$.

the PNI group, showed differences that approached significance only on Arithmetic $(p=0.10)$ and Block Counting $(p=0.07)$.

\section{Evidence of exacerbated decline 40 years after injury}

The significance of change from the 1950s to the 1980s (Table 3 ) was determined by paired-sample $t$ tests. PNI subjects improved over the 3 decades in mean Vocabulary score $(p<0.05)$, held steady in AGCT Total and Arithmetic, and declined in Block Counting and Hidden Figures $(p<0.05)$. In contrast, and regardless of locus of lesion, HI subjects as a group declined ( $p$ $\leq 0.01$ ) from the 1950 s to the 1980 s on every AGCT measure except Vocabulary, which was unchanged. Independent sample $t$-tests comparing change in the PNI and HI groups were significant $(p \leq 0.05)$ for the AGCT Total and Arithmetic measures (for Vocabulary, $p=0.07$ ), suggesting a general exacerbated decline effect among HI subjects regardless of lesion site.

Effects of site of injury. More detailed analyses sought effects of specific lesions on 1950s-to-1980s change. Separate ANOVAs were performed for the 8 lesion sites and 5 dependent measures, resulting in a total of 40 analyses, each of which compared HI subjects with a lesion at a specific site, HI subjects with no lesion at that site, and PNI subjects. No results are reported for the effect of temporal lobe injuries on performance of the Hidden Figures Test because of insufficient test-retest data for left and right temporal lobe groups.

One set of contrasts compared the change in test score of subjects in each of the 8 lesion groups to the change of subjects in the PNI group (e.g., left frontal HI versus PNI) (Table 4). For

\begin{tabular}{|c|c|c|c|c|c|c|}
\hline \multirow{2}{*}{$\frac{\text { Test }}{\text { AGCT Total }}$} & \multicolumn{2}{|c|}{ Impaired in $1950 \mathrm{~s}$} & \multicolumn{2}{|c|}{$\begin{array}{l}\text { Exacerbated decline } \\
1950 \text { s to } 1980 \mathrm{~s}\end{array}$} & \multicolumn{2}{|c|}{$\begin{array}{l}\text { Impaired in } 1950 \mathrm{~s} \\
\text { and exacerbated } \\
\text { decline }\end{array}$} \\
\hline & Left: & $\begin{array}{l}\text { temporal } \\
\text { parietal } \\
\text { occipital }\end{array}$ & $\begin{array}{l}\text { Left: } \\
\text { Right: }\end{array}$ & $\begin{array}{l}\text { parietal } \\
\text { occipital }\end{array}$ & & \\
\hline Vocabulary & Left: & $\begin{array}{l}\text { parietal } \\
\text { occipital }\end{array}$ & $\begin{array}{l}\text { Left: } \\
\text { Right: }\end{array}$ & $\begin{array}{l}\text { parietal } \\
\text { occipital }\end{array}$ & Left: & parietal \\
\hline Arithmetic & Left: & $\begin{array}{l}\text { temporal } \\
\text { parietal } \\
\text { occipital }\end{array}$ & Left: & $\begin{array}{l}\text { temporal } \\
\text { parietal }\end{array}$ & Left: & $\begin{array}{l}\text { temporal } \\
\text { parietal }\end{array}$ \\
\hline Block Counting & Left: & $\begin{array}{l}\text { temporal } \\
\text { parietal } \\
\text { occipital }\end{array}$ & & & & \\
\hline Hidden Figures & $\begin{array}{l}\text { Left: } \\
\text { Right: }\end{array}$ & $\begin{array}{l}\text { parietal } \\
\text { occipital } \\
\text { occipital }\end{array}$ & Right: & parietal & & \\
\hline
\end{tabular}




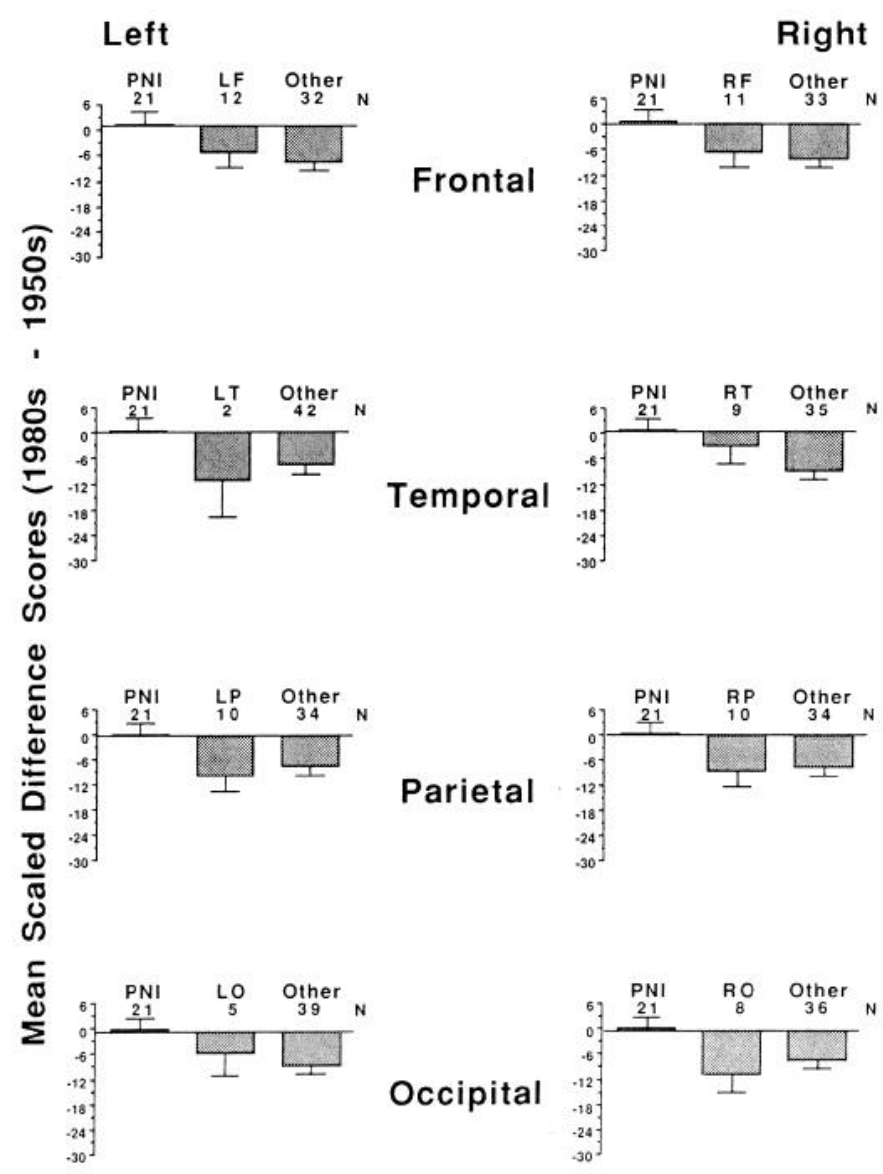

Figure 1. AGCT Total score. Each of the 8 graphs represents 1980 s1950s difference scores for the PNI group, the specified lesion group, and all other HI subjects. $L F$, left frontal; $R F$, right frontal; $L T$, left temporal; $R T$, right temporal; $L P$, left parietal; $R P$, right parietal; $L O$, left occipital; $R O$, right occipital.

the AGCT Total measure, the left parietal and right occipital groups declined more than the PNI group $(p<0.05)$ (Fig. 1). For the Vocabulary subtest, the left parietal and right occipital groups differed significantly from the PNI group $(p<0.05$ for each) (Fig. 2). For the Arithmetic subtest, injury to the left temporal $(p<0.01)$ and left parietal $(p<0.05)$ lobes was associated with greater decline than that in the PNI group (Fig. $3)$. Block Counting scores showed no significant differences between groups (Fig. 4). For the Hidden Figures Test, exacerbated decline relative to the PNI control subjects occurred following right parietal lobe injury ( $p=0.02$ ) (Fig. 5).

Another set of contrasts compared the 1950s-to-1980s change in subjects who had a specific lesion to the change in the remaining HI subjects (e.g., left frontal versus other HI) (Figs. 15). Such contrasts were significant for the right occipital group on AGCT Total and for the left temporal group on the Arithmetic test ( $p \leq 0.05$ for each), as well as for the right parietal group on Hidden Figures $(p=0.02)$. Each of these lesion groups declined more than subjects without the specified lesion.

ANOVAs of the effects of site of injury could not use Bonferroni or other techniques to control type I error rates because of the large number of combinations of lesion sites (8) and dependent measures (5). The fact that the HI and PNI groups as a whole differed significantly in extent of decline on 2 of the 5 measures and differed at the 0.07 level for a third measure

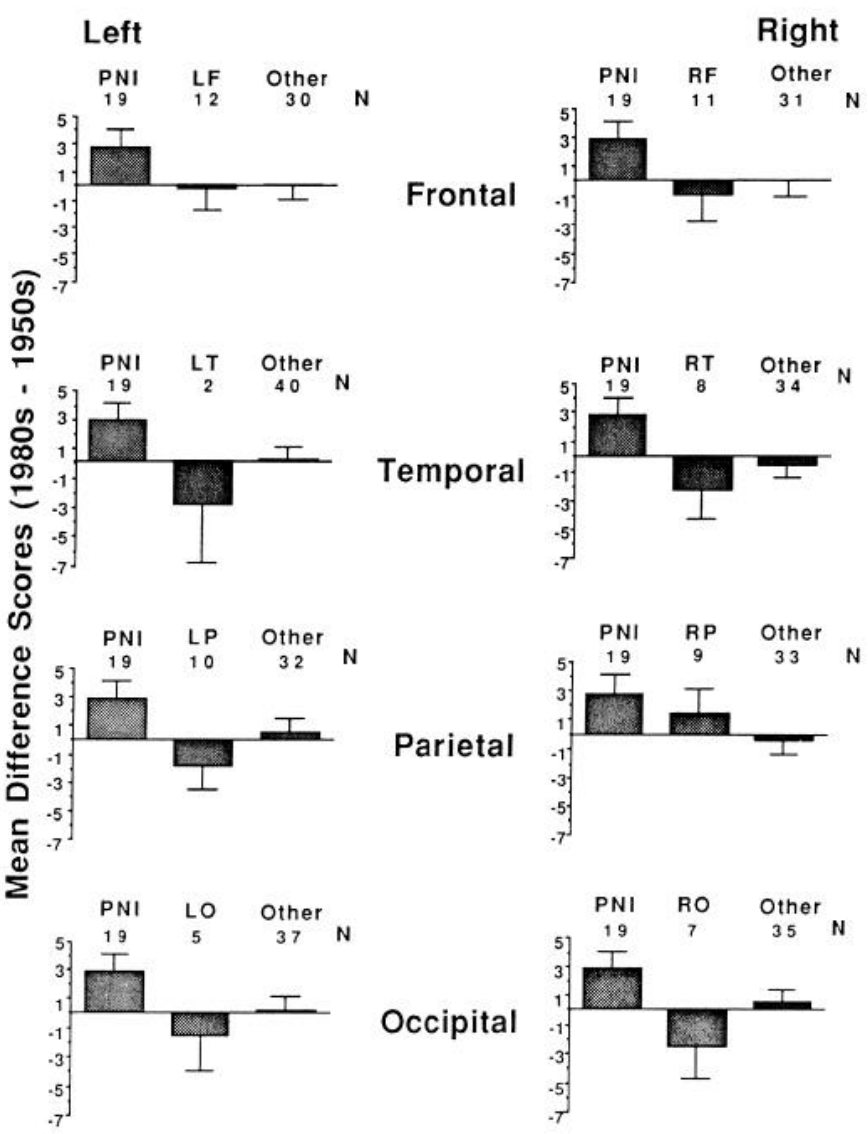

Figure 2. AGCT Vocabulary subtest. Each of the 8 graphs represents 1980s-1950s difference scores for the PNI group, the specified lesion group, and all other HI subjects. Abbreviations as in Figure 1.

indicates that the general exacerbated decline phenomenon is "real" rather than a type I error. The consistency of our results with current knowledge of brain-behavior relations also speaks to the results' validity. Left posterior lesions produced exacerbated decline on verbal tests, and right parietal lesions produced exacerbated decline on a spatial test. We cannot explain and thus are suspicious of the reproducibility of the relation between right occipital lesions and exacerbated decline on the Vocabulary subtest. Review of these subjects' charts (including information regarding lesion localization) failed to illuminate the observed finding.

Prevalence of exacerbated decline. The observed statistical significance of exacerbated decline does not speak to the issue of its prevalence, in particular, whether large declines in a few HI subjects accounted for the observed mean differences between the HI and PNI groups. We examined the distribution of 1980s-1950s differences in order to determine the percentage of HI subjects showing exacerbated decline, defined as declines greater than the median PNI change. Chi-square goodness of fit tests (2-tailed) determined whether significantly more than half the HI subjects showed such declines. Ignoring lesion site, the prevalence of exacerbated decline among HI subjects was $77.3 \%$ for AGCT Total scores $(p<0.001), 57.1 \%$ for Vocabulary $(n s)$, $70.7 \%$ for Arithmetic $(p<0.05), 46.3 \%$ for Block Counting $(n s)$, and $64.0 \%$ for Hidden Figures ( $n s$ ). (The $t$ tests comparing 1980s1950s changes in the HI and PNI groups also had revealed significant differences for AGCT Total and Arithmetic.) Indi- 


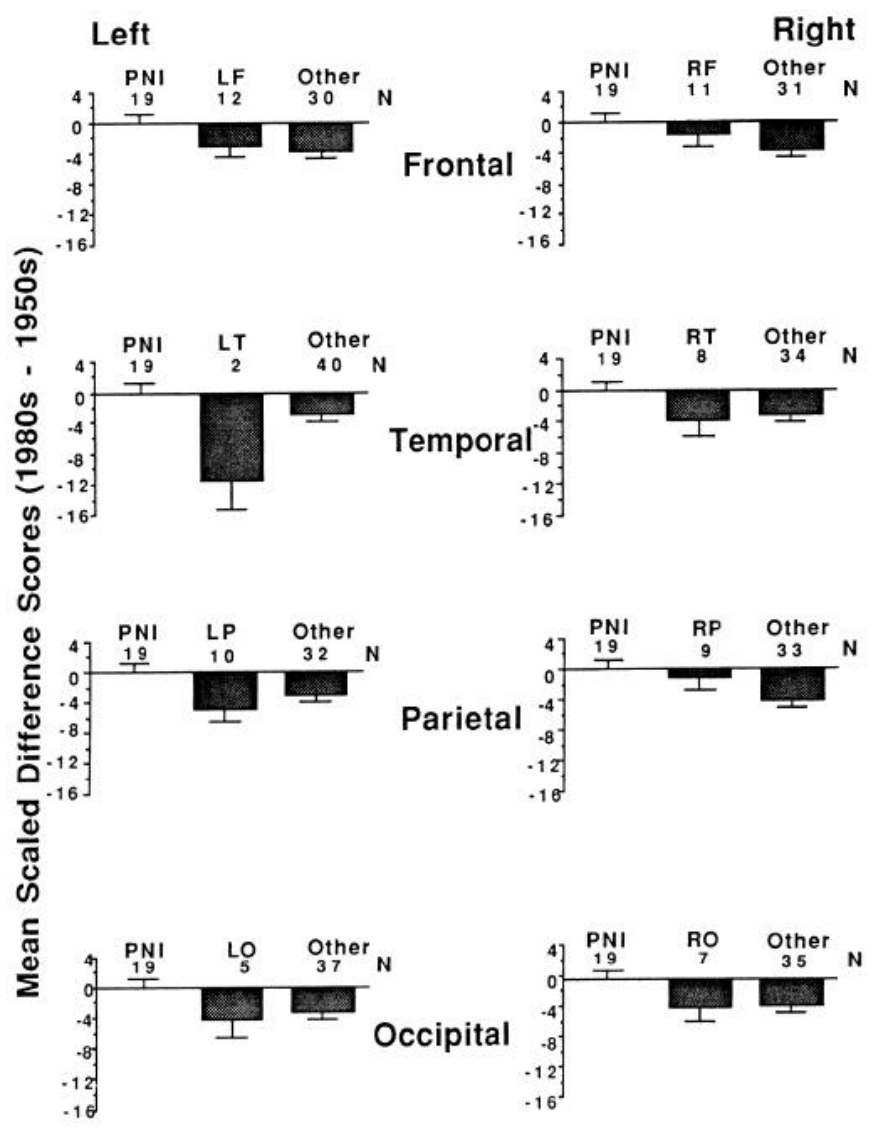

Figure 3. AGCT Arithmetic subtest. Each of the 8 graphs represents 1980s-1950s difference scores for the PNI group, the specified lesion group, and all other HI subjects. Abbreviations as in Figure 1.

vidual test-retest values for HI subjects are displayed in Figure 6. These graphs correspond to 4 of the combinations of lesion sites and cognitive tests for which $\mathrm{HI}$ subjects sustaining a particular injury showed significant mean declines relative to the PNI sample. As demonstrated by the chi-square tests and graphs, exacerbated decline was common rather than limited to a few HI subjects.

\section{Role of subjects' age and education}

We examined the correlations between subjects' age at injury and the degree of cognitive decline observed over the 30-year test-retest interval. Age at injury and age at testing were highly correlated in the 1950s $(r=0.94)$ and in the 1980s $(r=0.90)$, and therefore these variables were equivalent for predictive purposes. Additional analyses correlated years of education with cognitive decline over 30 years.

The correlations between age at injury and the 1980s-1950s differences were near zero for PNI subjects (Table 5). In contrast, within the HI sample, the difference scores for the AGCT Total, Vocabulary, and Block Counting measures correlated significantly with age at injury $(p<0.05)$ : the older the subject when injured or tested, the more performance declined from the 1950s to the 1980 s. These correlations largely reflected the relation between age and performance in the $1980 \mathrm{~s}$. Greater age at injury was associated with worse 1980 s performance $(p<0.05$ for AGCT Total and $p<0.01$ for Block Counting) and performance in the 1950 s was independent of age at injury. Unlike the HI
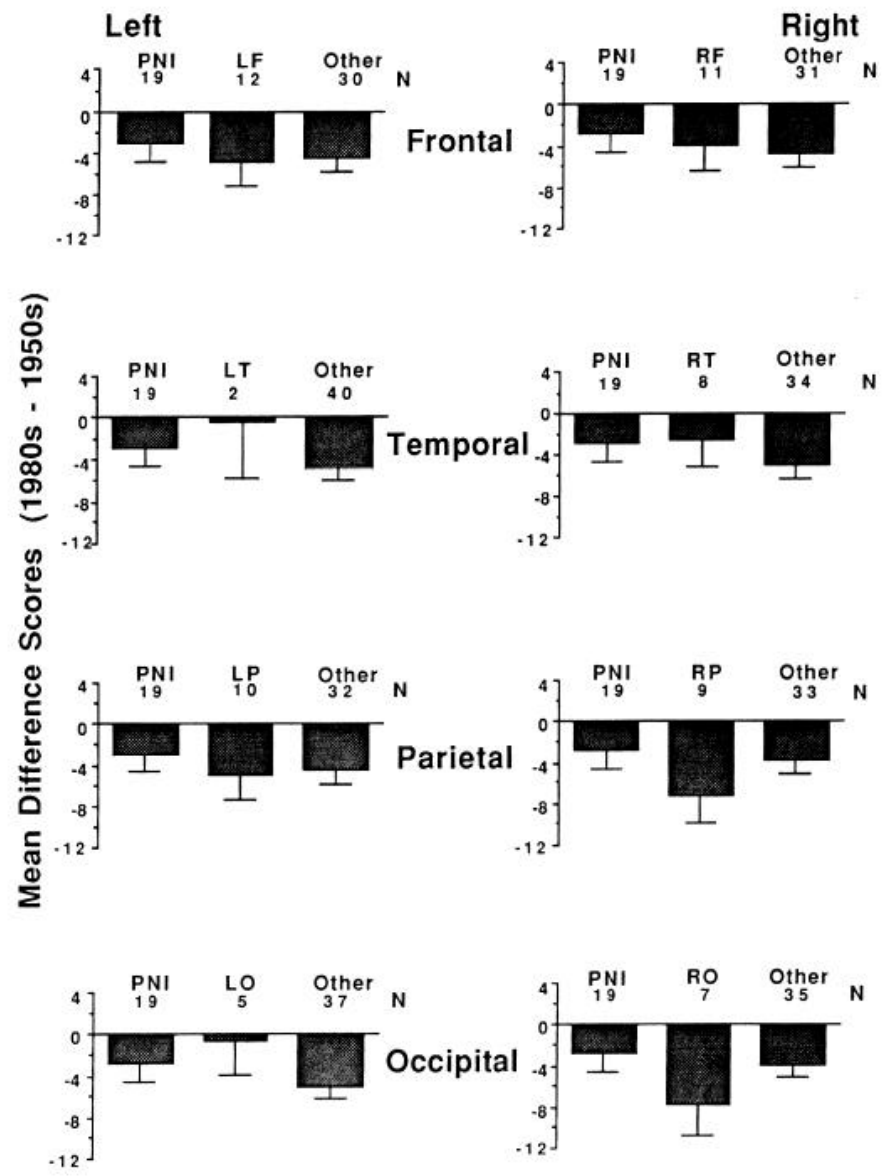

Figure 4. AGCT Block Counting subtest. Each of the 8 graphs represents 1980s-1950s difference scores for the PNI group, the specified lesion group, and all other HI subjects. Abbreviations as in Figure 1.

subjects, older PNI subjects scored better than younger subjects, demonstrating that the negative association between age and performance within the HI sample resulted from an age-injury interaction rather than from the effects of age alone.

There were no significant correlations between preinjury or postinjury education and 1980 s- 1950 s difference scores in either the HI or PNI groups, suggesting that exacerbated decline is not related to the subjects' intellectual level as indicated by years of education.

\section{Relation between severity of injury and cognitive measures}

The 4 measures of severity of $\mathrm{HI}$ that were available for the HI subjects were the number of cortical lobes involved in the injury (mean $=1.5)$, presence of a tantalum plate $(n=21)$, history of seizures $(n=21)$, and use of anticonvulsant medication $(n=$ 26). None of these variables predicted the magnitude of the 1950s-to-1980s cognitive changes. Within our sample, the occurrence of exacerbated decline depended on the site rather than the severity of injury.

\section{Discussion}

We observed exacerbated cognitive decline by comparing subjects with long-standing $\mathrm{HI}$ to control subjects with long-standing PNI. All were injured during World War II and were followed from the 1950 s until the $1980 \mathrm{~s}$. We found that penetrating 
HI was associated with exacerbated decline relative to PNI and that the effect was readily detectable on tests of cognitive function. For example, on the AGCT Total score, HI subjects lost an average of 7.9 points from the 1950 s to the $1980 \mathrm{~s}$, whereas PNI subjects gained an average of 0.4 points; the 8.3 point difference represents about $40 \%$ of an SD. Exacerbated decline was particularly pronounced for older subjects (either at injury or at test). The Discussion focuses on 2 questions about exacerbated decline: (1) How general is this phenomenon, and (2) what mechanisms could produce it?

It is doubtful that our observation of exacerbated decline among HI subjects resulted from selective dropouts. Sullivan and Corkin (1984) reported that HI subjects retested during the 1980s had more education and higher preinjury and 1950s AGCT Total scores than HI subjects tested only in the 1950 s, suggesting an association between impaired functioning and subject dropout. We do not know if exacerbated cognitive decline decreased the likelihood of subjects' returning for testing in the 1980s. Any such bias, however, would have diminished the magnitude of exacerbated decline.

\section{Generality of exacerbated decline}

The question of generality has 2 aspects. The first concerns the types of behavioral functions that are subject to exacerbated decline, and the second concerns the diversity of lesions that cause exacerbated decline.

Functions that show exacerbated decline. The present study of the aftermath of HI demonstrated exacerbated decline on a test of general intelligence and (among patients with right parietal lobe lesions) on a figure-ground discrimination task. Hamlin (1970) studied the aftermath of psychosurgery and found that superior topectomy exacerbated decline in Wechsler-Bellevue scores. When reexamined 8-14 years after operation, nonoperated control subjects showed little change in WechslerBellevue scores. Patients who underwent orbital topectomy showed significant gains, but patients who received superior topectomy experienced a drop in verbal test performance that was greater over the last 6 years of the 14-year period than over the first 8 years. Studies of the postpolio syndrome have found exacerbated decline of motor function; survivors of polio may experience new muscular symptoms many years after their recovery from acute paralytic poliomyelitis (Dalakas et al., 1986). We suspect that additional behavioral capacities may manifest exacerbated decline.

Evidence for generality. Exacerbated decline on the AGCT

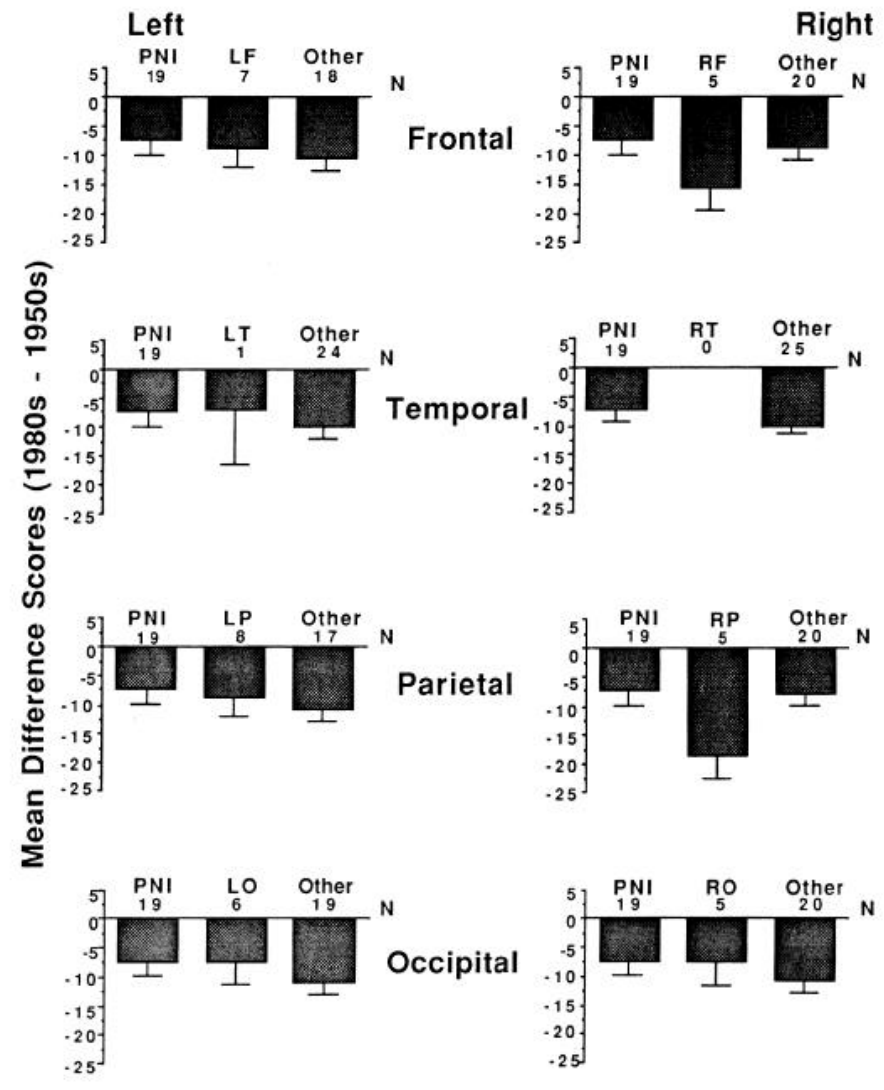

Figure 5. Hidden Figures Test. Each of the 8 graphs represents 1980s1950 s difference scores for the PNI group, the specified lesion group, and all other HI subjects. Abbreviations as in Figure 1.

test seemed a general consequence of HI. When only the direction of differences was considered, all 8 lesion groups declined more than the PNI subjects on AGCT Total, Vocabulary, and Arithmetic (Figs. 1-3).

Evidence for focality. If exacerbated decline depended on the specific locus of lesion, HI subgroups should have differed in the tests on which they manifested such decline. Evidence that decline depended on locus of lesion was seen in the performance losses for the left parietal group on the Vocabulary subtest, for the left temporal and parietal groups on the Arithmetic subtest, and for the right parietal group on the Hidden Figures Test. The behavioral measures of change were inevitably limited by the

Table 5. Correlations between age at injury and test performance

\begin{tabular}{|c|c|c|c|c|c|c|c|c|}
\hline \multirow[b]{2}{*}{ Test } & \multicolumn{4}{|c|}{ HI group } & \multicolumn{4}{|c|}{ PNI group } \\
\hline & $n$ & $1950 \mathrm{~s}$ & $1980 \mathrm{~s}$ & $\begin{array}{l}1980 \mathrm{~s}- \\
1950 \mathrm{~s} \\
\text { differences }\end{array}$ & $n$ & $1950 \mathrm{~s}$ & $1980 \mathrm{~s}$ & $\begin{array}{l}\text { 1980s- } \\
1950 \text { s } \\
\text { differences }\end{array}$ \\
\hline AGCT Total & 44 & -0.13 & $-0.34^{b}$ & $-0.34^{b}$ & 21 & 0.32 & 0.31 & -0.07 \\
\hline Vocabulary & 42 & -0.05 & -0.21 & $-0.30^{b}$ & 19 & $0.43^{a}$ & $0.48^{b}$ & -0.06 \\
\hline Arithmetic & 42 & -0.05 & -0.14 & -0.18 & 19 & 0.35 & 0.19 & -0.22 \\
\hline Block Counting & 42 & $-0.25^{a}$ & $-0.44^{c}$ & $-0.30^{b}$ & 19 & 0.24 & $0.39^{a}$ & 0.16 \\
\hline Hidden Figures & 25 & -0.26 & -0.21 & 0.11 & 14 & 0.14 & 0.14 & -0.02 \\
\hline
\end{tabular}

${ }^{a} p \leq 0.10$.

${ }^{b} p \leq 0.05$.

$c p \leq 0.01$. 


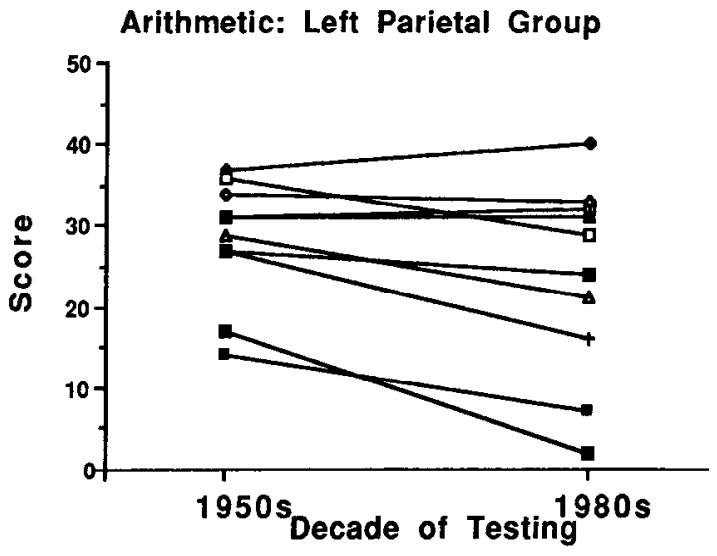

Vocabulary: Left Parietal Group

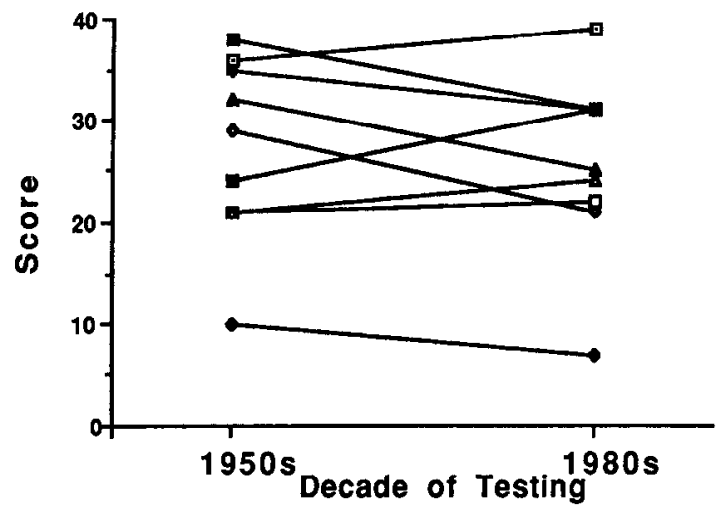

Arithmetic: Left Temporal Group

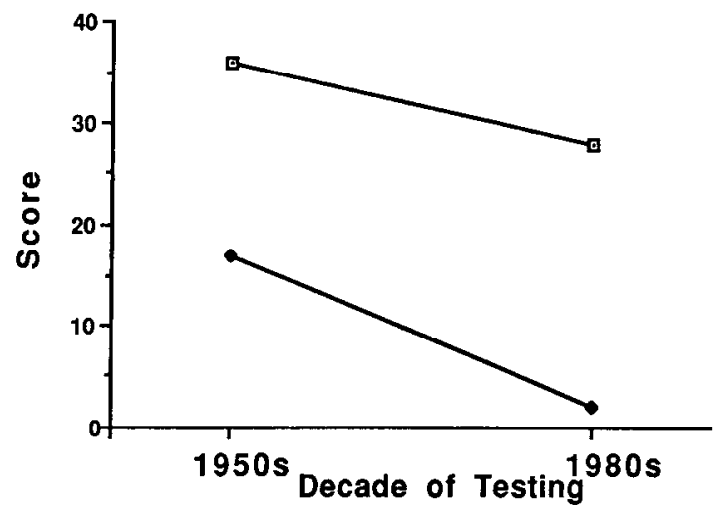

Hidden Figures: Right Parietal Group

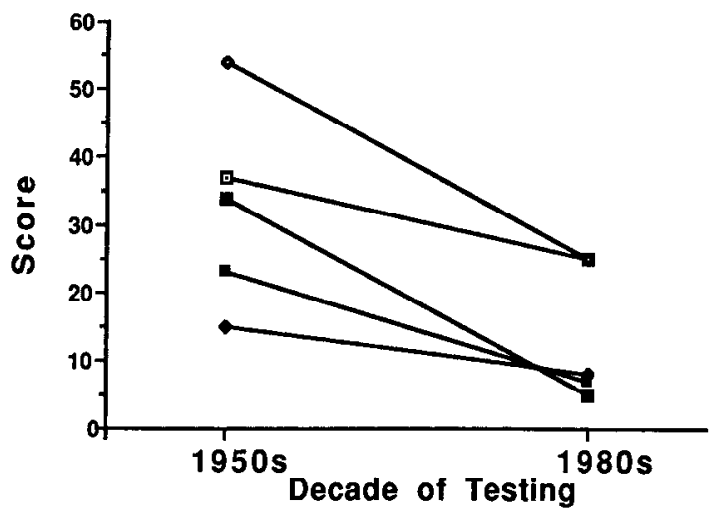

Figure 6. Cognitive changes for individual HI subjects. Within cach graph, subjects with the indicated lesion showed significant mean exacerbated decline $(p<0.05)$ relative to PNI subjects on the indicated cognitive measure.

lack of systematic data on other tests in the 1950s and the 1980s for the same patients. Additional focal effects might have been observed if subjects had received additional tests, e.g., tests sensitive to frontal lobe dysfunction or memory impairment.

Because Teuber and Weinstein (1956) found that HI subjects were significantly inferior in Hidden Figures performance relative to PNI subjects, we did not expect that the Hidden Figures Test would prove the most "focal" measure in our study (i.e., the most dependent on a single brain region). Our finding that only the right parietal group showed exacerbated decline from the 1950 s to the 1980 s on Hidden Figures, together with Teuber and Weinstein's findings of a correspondence between poor performance and aphasia (implicating a left parietal lobe lesion in at least some of the cases), suggests that the left and right parietal cortices each contribute to successful performance on this task. Our previous study of Korean Conflict veterans tested 20 years after HI supported this view (Corkin, 1979). Regardless of side, lesions in the vicinity of the middle cerebral artery were associated with deficits on the Hidden Figures Test.

Conditions producing exacerbated decline. In part, studying the generality of exacerbated decline means identifying the conditions under which it occurs. Exacerbated decline seems to depend on the nature of the brain injury, the age at injury, and the time interval between injury and testing, i.e., the same factors that determine the specific consequences of brain injury (Schoenfeld and Hamilton, 1977).
Other studies as well as our own suggest that exacerbated cognitive decline following $\mathrm{HI}$ becomes observable around or shortly after age 50 . Our subjects had a mean age of 62 at followup, at which time exacerbated decline effects were pronounced. Walker (1972) evaluated 195 veterans who had sustained HI in World War II and experienced posttraumatic seizures. At follow-up (mean age $=50$ years), veterans who reported greater progressive neurological deficit were more likely to be judged by their spouses as aging unduly rapidly than were veterans whose neurological deficit had not changed. Deterioration had not been evident in these men 10 years earlier (Walker and Erculei, 1969), implying that exacerbated decline began between ages 40 and 50. Future studies should address issues related to the time course of exacerbated decline.

\section{Biological substrates of exacerbated decline}

Explanations of exacerbated decline based on the margin-ofsafety model attribute behavioral deficits to the effects of neural degeneration associated either with aging or with the brain's response to the original injury. The margin-of-safety model proposes that the brain's organization is inherently redundant, that a substantial amount of tissue can be lost without apparent consequences (Teuber, 1974; Glassman, 1987), that HI decreases redundancy, that aging and the secondary effects of $\mathrm{HI}$ also decrease redundancy, and that the cumulative loss of re- 
dundancy may cause the appearance of new deficits or exacerbate existing deficits years after the initial injury.

The fact that age and performance correlated only among HI subjects suggests that age-related factors contributed to exacerbated decline. The high correlation between age at injury and at testing leaves open the question of whether the age-performance association reflects subjects' age-related condition of the brain at the time of injury or the relation between subjects' age at testing and the age at which behavioral decline occurs most rapidly. Any or all of several mechanisms could result in neuronal loss or degradation after the initial injury, including aging, transneuronal degeneration secondary to HI (Cowan, 1970), and the brain's reaction to the additional stress placed on it by having to function for the years following injury in a compromised state (Cotman and Nietro-Sampedro, 1984; Flood et al., 1987). Neuronal death or atrophy may continue years after the original injury (Geschwind, 1974). For example, Yakovlev (1953) observed degenerative changes in the brains of patients who had undergone frontal lobotomy and who survived thereafter from 2 weeks to $4 \frac{1}{2}$ years. Patients with longer survival periods had smaller and more atrophic brains than those with shorter survival periods. Because postinjury degeneration may cause widespread changes in the central nervous system, it could explain the exaccrbated cognitive decline documented in the present report.

Our data support the margin-of-safety model in some respects but not in others. The observation of exacerbated decline in already defective capacities is consistent with the model. Four of the 7 instances of exacerbated decline occurred in groups that had been impaired on the same measures in the 1950 s, 10 years after injury (Table 4). Inconsistent with the model is the absence of exacerbated decline in Block Counting in 3 lesion groups that had shown impairments in the 1950s, and the combination of 1950s impairment in Hidden Figures performance within the left parietal group and exacerbated decline within the right parietal group. Unpredicted by the margin-of-safety model was an apparent tradeoff between lesion and aging effects. The HI group showed significant exacerbated decline on the tasks most resistant to aging among PNI subjects (AGCT Total and Arithmetic; see Table 3). The PNI group's decline over time on the Block Counting and Hidden Figures measures fits prior observations that cognitive loss becomes apparent among normal subjects sometime after age 60 , particularly on tasks that require some type of novel cognitive activity, e.g., perceptual reasoning or pattern analysis (Cunningham and Owens, 1983) and active intellectual abilities (Schaie, 1983). We conclude that a satisfactory explanation of the delayed effects of HI thus requires integrating aging effects and task demands.

\section{Diminished experience}

Exacerbated decline in function in HI subjects may not be due solely to abnormal brain function but may also be attributable to a decline in socioeconomic status or to other aspects of life experiences since the time of injury. Possible psychosocial factors influencing cognition include education, autonomy, and social support (Rowe and Kahn, 1987), as well as intellectual, vocational, recreational, and stressful experiences.

\section{Clinical significance}

We encourage clinicians, before making a diagnosis of dementia in an HI patient, to consider the possibility that exacerbated cognitive decline rather than a separate pathological process may explain newly emergent deficits. Although none of our HI subjects was demented when tested in the $1980 \mathrm{~s}$, it is possible that continuing rapid decline will result in dementia at some later date. We are, therefore, continuing our follow-up study of the HI and PNI groups in order to detect possible global decline.

\section{References}

Corkin, S. (1979) Hidden-figures-test performance: Lasting effects of unilateral penetrating head injury and transient effects of bilateral cingulotomy. Neuropsychologia 17: 585-605.

Cotman, C. W., and M. Nieto-Sampedro (1984) Cell biology of synaptic plasticity. Science 225: 1287-1294.

Cowan, W. M. (1970) Anterograde and retrograde transneuronal degeneration in the central and peripheral nervous system. In Contemporary Research Methods in Neuroanatomy, W. J. H. Nauta and S. O. E. Ebbesson, eds., pp. 217-251, Springer-Verlag, New York.

Cunningham, W. R., and W. A. Owens, Jr. (1983) The Iowa state study of the adult development of intellectual abilities. In Longitudinal Studies of Adult Psychological Development, K. W. Schaie, ed., pp. 20-39, Guilford, New York.

Dalakas, M. C., G. Elder, M. Hallett, J. Ravits, M. Baker, N. Papadopoulos, P. Albrecht, and J. Sever (1986) A long-term follow-up study of patients with post-poliomyelitis neuromuscular symptoms. N. Engl. J. Med. 314: 959-963.

Flood, D. G., S. J. Buell, G. J. Horwitz, and P. D. Coleman (1987) Dendritic extent in human dentate gyrus granule cells in normal aging and senile dementia. Brain Res. 402: 205-216.

Geschwind, N. (1974) Late changes in the nervous system: An overview. In Plasticity and Recovery of Function in the Central Nervous System, D. G. Stein, J. J. Rosen, and N. Butters, eds., pp. 467-508, Academic, New York.

Glassman, R. B. (1987) An hypothesis about redundancy and reliability in the brains of higher species: Analogies with genes, internal organs, and engineering systems. Neurosci. Biobehav. Rev. 11: 275285.

Hamlin, R. M. (1970) Intellectual function 14 years after frontal lobe surgery. Cortex 6: 299-307.

Personnel Research Section, Classification and Enlisted Replacement Branch, The Adjutant General's Office (1943) Personnel Research in the Army. I. Background and organization. Psychol. Bull. 40: 129135.

Rowe, J. W., and R. L. Kahn (1987) Human aging: Usual and successful. Science 237: 143-149.

Schaie, K. W. (1983) The Seattle longitudinal study: A 21-year exploration of psychometric intelligence in adulthood. In Longitudinal Studies of Adult Psychological Development, K. W. Schaie, ed., pp. 64-135, Guilford, New York.

Schoenfeld, T. A., and L. W. Hamilton (1977) Secondary brain damages following lesions: A new paradigm for lesion experimentation. Physiol. Behav. 18: 951-967.

Semmes, J., S. Weinstein, L. Ghent, and H.-L. Teuber (1960) Somatosensory Changes After Penetrating Brain Wounds in Man, Harvard U. P., Cambridge, Mass.

Sullivan, E. V., and S. Corkin (1984) Selective subject attrition in a longitudinal study of head-injured veterans. J. Gerontol. 39: 718720 .

Teuber, H.-L. (1974) Recovery of function after lesions of the central nervous system: History and prospects. Neurosci. Res. Program Bull. 12: 197-211.

Teuber, H.-L., and S. Weinstein (1956) Ability to discover hidden figures after cerebral lesions. Arch. Neurol. Psychiatry 76: 369-379.

Thurstone, L. L. (1944) A Factorial Study of Perception, University of Chicago Press, Chicago.

Walker, A. E. (1972) Long-term evaluation of the social and family adjustment in head injuries. Scand. J. Rehabil. Med. 4: 5-8.

Walker, A. E., and F. Erculei (1969) Head Injured Men: Fifteen Years Later, Charles C Thomas, Springfield, Ill.

Wechsler, D. (1981) WAIS-R Manual: Wechsler Adult Intelligence Scale, Revised, Harcourt Brace Jovanovich, New York.

Weinstein, S., and H.-L. Teuber (1957) Effects of penetrating brain injury on intelligence test scores. Science 125: 1036-1037.

Yakovlev, P. I. (1953) Fronto-pontine bundle and associated projection fibers of the frontal lobe following frontal leucotomy. Trans. Am. Neurol. Assoc. 78: 286-291. 